\title{
Programa permanente de análisis de la demanda turística del Puyo, observatorio turístico de la Universidad Estatal Amazónica
}

\section{Permanent program of analysis of the tourist demand of Puyo of tourist observatory of the Amazon State University}

Mayra Pinta Rodríguez. ${ }^{1}$, Dunia Chávez Esponda. ${ }^{2}$ \& Eduardo Ruiz Mármol. ${ }^{3}$

\begin{abstract}
.
DOI: https://doi.org/10.33262/cienciadigital.v3i3.651

Communication is essential for social change, respecting languages and history, creating a communicative identity that looks to the future in a proposal based on dialogue. So since 2015, Ecuador has used a strategic tool that allows it to update tourism statistics by means of the economic aspect. In the Amazonian region, the scarce and informal statistical information of the tourist demand, that support the decision making, led to the Amazon State University implementing the Tourist Observatory, denominated with acronyms OT-UEA. The present research was based on the Management Model of the OT-UEA, to create the Permanent Program for the Analysis of Tourism Demand. The methodology applied to knowledge searching was scientific observation as a method, which consisted of direct observation expressed by the perceptions of visitors who came to the different tourist attractions of the city of Puyo. The Inductive - Deductive method was also used to identify the main variables and the survey technique of the research was structured and organized for the collection of information, aimed at all visitors who arrived at the most popular tourist attractions. Finally, with the data obtained it was possible to know the tourist demand (profile of the visitors) and to design the first newsletter OT-UEA 2018 for its diffusion, which will serve as an instrument for decision making of the actors within the tourism system.
\end{abstract}

Key Words: Program, Tourist Demand, Puyo, Tourist Observatory.

\section{Resumen.}

\footnotetext{
${ }^{1}$ Universidad Estatal Amazónica. Pastaza, Ecuador, mpinta@uea.edu.ec

${ }^{2}$ Universidad Estatal Amazónica. Pastaza, Ecuador, dchavez@uea.edu.ec

${ }^{3}$ Universidad Estatal Amazónica. Pastaza, Ecuador, eruiz@uea.edu.ec.
} 
La comunicación es indispensable para el cambio social, respetando las lenguas y la historia, gestando una identidad comunicativa que mire al futuro un estilo basado en el diálogo. Es por ello que Ecuador desde el año 2015 utiliza una herramienta estratégica que le permita actualizar las estadísticas del turismo a través del aspecto económico. En la región Amazónica la escasa e informal información estadística de la demanda turística que respalden la toma de decisiones originó que la Universidad Estatal Amazónica implemente el Observatorio Turístico denominado con siglas OTUEA. La presente investigación, se amparó en el Modelo de Gestión del OT-UEA, para crear el Programa Permanente del Análisis de la Demanda Turística. La metodología que se aplicó para la búsqueda de conocimientos fue la observación científica como método, que consistió en la observación directa expresada por las percepciones de los visitantes que acudieron a los diferentes atractivos turísticos de la ciudad de Puyo. También se utilizó el método Inductivo - Deductivo, para identificar las principales variables y la técnica mediante la cual se estructuró y organizó la investigación para la recolección de información fue la encuesta, dirigida a todos los visitantes que llegaron a los atractivos turísticos más concurridos. Finalmente, con los datos obtenidos se pudo conocer la demanda turística (perfil de los visitantes) y realizar el diseño del primer boletín Informativo OT-UEA 2018 para su difusión, mismo que servirá de instrumento para toma de decisiones de los actores dentro del sistema turístico.

Palabras Clave: Programa, Demanda Turística, Puyo, Observatorio Turístico.

\section{Introducción}

Ecuador en el año 2015 implementó la Cuenta Satélite de Turismo convirtiéndose en uno de los 60 países en disponer de esta herramienta estratégica bajo las normas internacionales y metodologías propuestas por la Organización Mundial del Turismo (OMT) que permite un progreso en la actualización de las estadísticas del turismo en el aspecto económico; a través, de la medición de los bienes y servicios adquiridos por los visitantes en los diferentes sectores (Ministerio de Turismo, 2015).

La OMT (2017) señaló que, debido al incremento de destinos turísticos a nivel mundial, recibió de los Estados Miembros solicitudes de asistencia estadística con la finalidad de desarrollar información de los tipos de visitantes para la toma de decisiones; conservando el medio ambiente y la cultura del patrimonio; convirtiendo al turismo en el causal del crecimiento sostenible e inclusivo.

En la Región Amazónica, debido a la escasa e informal información estadística de la llegada de turistas, el comportamiento de la demanda, el asesoramiento en proyectos de inversión, la oferta instalada, entre otros factores que respaldan la toma decisiones; la UEA implementó el Observatorio Turístico (OT-UEA) en el año 2011, con el fin de entregar proyecciones socioeconómicas, estudios e investigaciones, formación por competencias y asistencia 
técnica en la gestión de negocios turísticos, para potenciar la competitividad del sector turístico en la Región Amazónica; así se cita en su Modelo de Gestión (Rangel, UEA 2013). Entre los objetivos estratégicos de la UEA (2018), estuvo contribuir al desarrollo local, regional y nacional generando nexos entre la Universidad y la Sociedad, a través de planes con nuevas alternativas que ayuden a solucionar problemas sociales y tecnológicos para fomentar la conservación de la naturaleza. Se ha considerado al OT-UEA como un eje de gestión de la UEA, a su personal académico y estudiantil para recolectar, procesar, analizar y difundir información confiable que servirá de herramienta para el desarrollo turístico, su modernización y potenciación de nuevos instrumentos que permitan estar a la vanguardia de los retos de la actualidad, permitiendo acceder los posibles visitantes a las diferentes ofertas (Ascanio, 2012; Centro de Estudios Superiores en Turismo, 2012).

La presente investigación, amparada en el Modelo de Gestión del OT-UEA, presenta el Programa Permanente del Análisis de la Demanda Turística, diseñado exclusivamente para la ciudad de Puyo, iniciado en marzo de 2018 con la fase administrativa y metodológica, en abril con la recopilación de información, culminando en la implementación y publicación del primer boletín en septiembre 2018 que se encuentra actualmente disponible para toda la colectividad. La implementación de un Programa Permanente de Análisis de la Demanda Turística permite generar información estadística suficiente, formal y constante con la intención de orientar a los actores de la actividad turística en sus decisiones (Boullón, 2006).

\section{Materiales y Métodos}

Ecuador se encuentra ubicado al Sur del continente americano, está conformado por 24 provincias entre ellas Pastaza, cuya extensión es de $29520 \mathrm{Km}^{2}$, una altura de $930 \mathrm{msnm}$, clima tropical húmedo que circunda los $20^{\circ} \mathrm{C}$. Su capital es Puyo, una ciudad con diversidad de flora y fauna es generadora de varios atractivos turísticos.

La investigación tuvo un diseño no experimental, de tipo descriptivo, transversal y correlacional, que permitió la recolección de la información de primera línea de las variables de acuerdo a la demanda y la entrega de reportes que alimentarán el OT-UEA. El enfoque investigativo fue cuantitativo y cualitativo. Se establecieron las competencias de los organismos de la superestructura turística en relación de la demanda aplicando un análisis descriptivo, realizando una identificación de involucrados, estudiando la normativa vigente y sistematizando la información.

El Programa Permanente se diseñó en tres fases: 1. Modelo administrativo y operativo del programa con la estructura organizativa, orgánico funcional y procesos fundamentales; 2. Diseño estadístico en función de los requerimientos de información y considerando los indicadores que debe levantar el OT-UEA. Se estructuró la encuesta que recogió las necesidades y permitió realizar un análisis cuantitativo de la demanda turística; 3 . Esbozo y publicación del boletín trimestral digital. 
Para su implementación se puso en funcionamiento el Programa Permanente propuesto a partir del sexto mes del año 2018 realizando las siguientes actividades: 1.Se asignó los responsables del proceso por el orgánico funcional, 2.Se validó la herramienta de recopilación de información utilizando la muestra a partir de población infinita, 3.Se elaboró el diagrama de intervención en territorio (encuestas), 4. Se realizó el trabajo de campo de aplicación de encuestas, 5. Se publicó el boletín Informativo Trimestral OT-UEA 2018 con la información sistematizada.

Los datos primarios de la información turística, se recolectaron a través de encuestas, emitidas por autoridades, prestadores de servicios turísticos, turistas involucrados en la actividad turística del Puyo. La información secundaria fue recolectada de documentos (libros, revistas, tesis, páginas web). Se utilizó un muestreo probabilístico (aleatorio) orientado al segmento de visitantes al Puyo. La muestra quedó conformada por 385 encuestas dirigidas a los visitantes. Esta muestra fue considerada en cada mes de la investigación (junio, julio y agosto 2018) dando un total de 1.155 encuestados.

Para analizar la validez del cuestionario se utilizó el Alfa de Cronbach. Se utilizaron Tablas de Contingencia, Pruebas Chi-cuadrado y Coeficientes de Contingencia para ver la relación significativa entre las variables cualitativas. Se tomó un nivel de significación del 5\% y confiabilidad del 95\%. Las variables relacionadas entre sí, se usaron para la elaboración de las tablas personalizadas que contribuyeron a definir la demanda turística en los meses analizados. Los datos fueron procesados utilizando el software estadístico SPSS, versión 22.0. Finalmente, para el diseño del primer boletín se generaron doce grupos de variables de relevancia para esta investigación, mismos que constituyeron la base de la publicación.

\section{Resultados y Discusión}

La estructura organizativa para el Programa Permanente del OT-UEA referido a la Demanda Turística de Puyo, se muestra a continuación (Figura 1):

Figura 1: Estructura organizativa para el programa permanente del OT-UEA

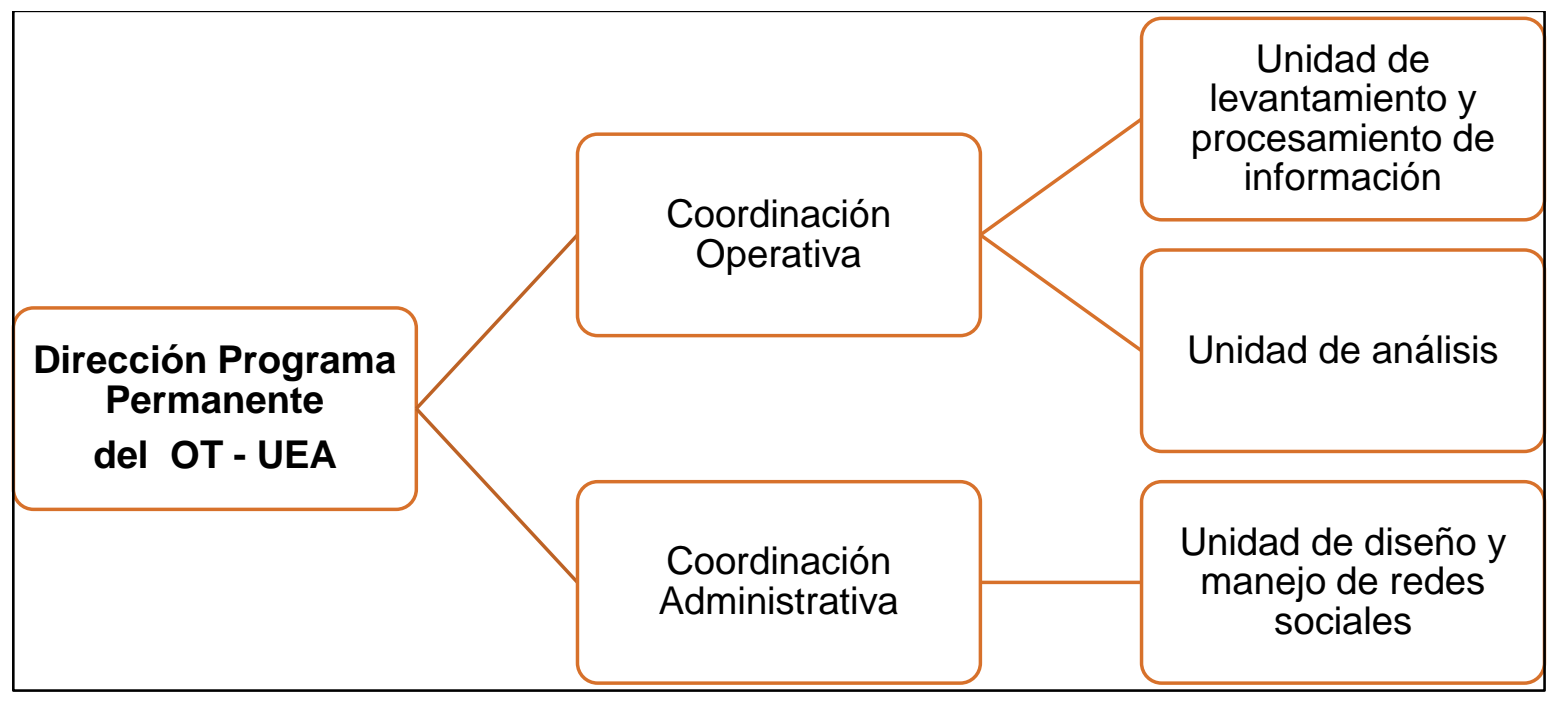


Dirección del Programa Permanente del OT-UEA: Dirigido por un profesional con título de cuarto nivel y Economista de profesión, con experiencia en el diseño de estudios económicos y con un amplio conocimiento de economía, econometría, estadística, investigación de mercados y mercadeo de servicios. Sus funciones serán: Dirigir y coordinar administrativa y operativamente el Programa Permanente del OT-UEA, encargándose de toda la información que surge de la actividad turística y estructurar y diseñar el perfil requerido para el equipo administrativo y operativo.

Coordinación Operativa del Programa Permanente del OT-UEA: Conformada por un profesional Economista, Ingeniero Comercial, Ingeniero en Estadística, o Ingeniero en Turismo con un amplio conocimiento de economía, econometría, estadística, investigación de mercados y mercadeo de servicios. Sus funciones serán: Generar información confiable de la demanda turística para la toma de decisiones de los diferentes actores turísticos y entregar una información trimestral a través de un boletín del análisis de la demanda turística en la ciudad de Puyo. Dentro de esta coordinación encontramos dos unidades:

Coordinación Administrativa del Programa Permanente del OT-UEA: Conformada por un profesional Economista, Ingeniero Comercial, Ingeniero en Estadística, o Ingeniero en Turismo con un amplio conocimiento en administración, finanzas, proyectos, estadística, investigación de mercados y mercadeo de servicios. Sus funciones serán: Coordinar el proceso metodológico del diseño, difusión, manejo de las redes sociales, proyectos y capacitación y emitir directrices para la evaluación de proyectos de inversión en el sector turístico. Esta coordinación cuenta con una unidad que a continuación se detalla:

Los resultados del análisis de fiabilidad para verificar la validez estadística de la encuesta mostraron un Alfa de Cronbach con un valor de 0,962 lo cual confirma la fiabilidad y validez del cuestionario ya que dicho valor es muy cercano a 1. Luego las escalas consideradas en las variables de estudio fueron excelentes según criterios de George \& Mallery (2003), quienes plantean que un coeficiente Alfa de Cronbach superior a 0.9 se considera excelente.

Luego de haber validado la fiabilidad del instrumento, se analizó la relación estadísticamente significativa entre las variables en estudio con las Tablas de contingencia, Pruebas de hipótesis Chi-cuadrado y Coeficientes de contingencia. Se consideraron las variables según el objetivo de la investigación y los indicadores que se mencionaron en el OT-UEA para la medición de la demanda turística: Género, Edad, Ciudad_País, Atractivo turístico, Gasto turístico, Ingresos, Medios de Información, Motivo de visita, Noches de estadía, Idea de la ciudad y Tipo de alojamiento. El análisis de la relación estadísticamente significativa entre las variables antes mencionadas se muestra a continuación, considerando un nivel de significación del 5\% y un nivel de confiabilidad del 95\% (Tablas 1, 2 y 3). 
Tabla 1: Resultados prueba Chi-cuadrado y Coeficiente de contingencia, datos de junio 2018

\begin{tabular}{|c|c|c|c|c|c|c|c|}
\hline \multicolumn{8}{|c|}{ MES JUNIO 2018} \\
\hline \multirow[b]{2}{*}{$\mathbf{N}^{\circ}$} & \multirow{2}{*}{$\begin{array}{c}\text { Combinación } \\
\text { de variables }\end{array}$} & \multirow{2}{*}{ Variables comparadas } & \multicolumn{3}{|c|}{ Chi-cuadrado de Pearson } & \multicolumn{2}{|c|}{$\begin{array}{l}\text { Coeficiente de } \\
\text { contingencia }\end{array}$} \\
\hline & & & Valor & Gl & $\begin{array}{c}\text { Sig. } \\
\text { asintótica } \\
\text { ( } 2 \text { caras })\end{array}$ & Valor & $\begin{array}{c}\text { Aprox. } \\
\text { Sig. }\end{array}$ \\
\hline & \multirow{36}{*}{ 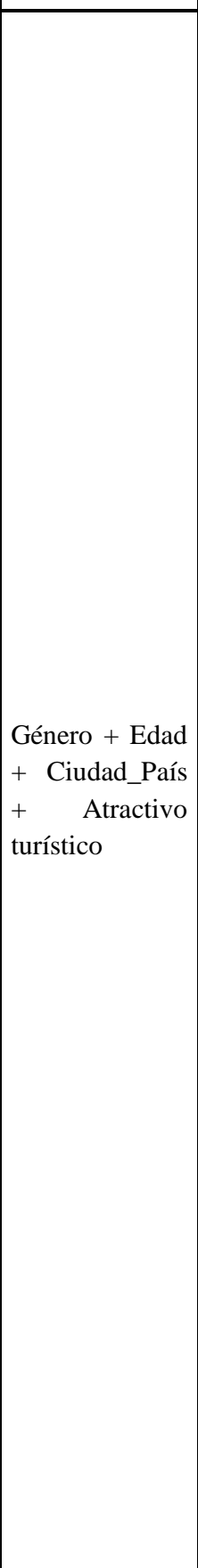 } & Género + Parque Acuático Morete Puyu & 9,190 & 1 & 0,002 & 0,453 & 0,002 \\
\hline & & Edad + Parque Acuático Morete Puyu & 9,586 & 5 & 0,009 & 0,559 & 0,009 \\
\hline & & Ciudad_País + Parque Acuático Morete Puyu & 82,762 & 27 & 0,000 & 0,421 & 0,000 \\
\hline & & Género + Parque Central Doce de Mayo & 8,752 & 1 & 0,003 & 0,491 & 0,003 \\
\hline & & Edad + Parque Central Doce de Mayo & 25,108 & 5 & 0,000 & 0,474 & 0,000 \\
\hline & & Ciudad_País + Parque Central Doce de Mayo & 125,700 & 27 & 0,000 & 0,496 & 0,000 \\
\hline & & Género + Museo Etnoarqueológico de Pastaza & 8,301 & 1 & 0,004 & 0,453 & 0,004 \\
\hline & & Edad + Museo Etnoarqueológico de Pastaza & 7,000 & 5 & 0,022 & 0,434 & 0,022 \\
\hline & & Ciudad_País + Museo Etnoarqueológico de Pastaza & 27,966 & 27 & 0,041 & 0,426 & 0,041 \\
\hline & & Género + Refugio de Vida Silvestre Yana Cocha & 3,526 & 1 & 0,006 & 0,495 & 0,006 \\
\hline & & Edad + Refugio de Vida Silvestre Yana Cocha & 10,336 & 5 & 0,007 & 0,617 & 0,007 \\
\hline & & Ciudad_País + Refugio de Vida Silvestre Yana Cocha & 158,812 & 27 & 0,000 & 0,540 & 0,000 \\
\hline & & Género + Paseo turístico del Río Puyo & 0,001 & 1 & 0,010 & 0,540 & 0,010 \\
\hline & & Edad + Paseo turístico del Río Puyo & 11,468 & 5 & 0,043 & 0,701 & 0,043 \\
\hline & & Ciudad_País + Paseo turístico del Río Puyo & 91,824 & 27 & 0,000 & 0,439 & 0,000 \\
\hline & & Género + Malecón Boayaku Puyu & 3,152 & 1 & 0,008 & 0,901 & 0,008 \\
\hline & & Edad + Malecón Boayaku Puyu & 13,036 & 5 & 0,023 & 0,481 & 0,023 \\
\hline & & Ciudad_País + Malecón Boayaku Puyu & 136,241 & 27 & 0,000 & 0,511 & 0,000 \\
\hline & & Género + Parque Etnobotánico Omaere & 6,098 & 1 & 0,014 & 0,487 & 0,014 \\
\hline & & Edad + Parque Etnobotánico Omaere & 13,313 & 5 & 0,021 & 0,483 & 0,021 \\
\hline & & Ciudad_País + Parque Etnobotánico Omaere & 156,432 & 27 & 0,000 & 0,538 & 0,000 \\
\hline & & Género + Jardín Botánico Las Orquídeas & 6,098 & 1 & 0,014 & 0,487 & 0,014 \\
\hline & & \begin{tabular}{|l} 
Edad + Jardín Botánico Las Orquídeas \\
\end{tabular} & 17,461 & 5 & 0,004 & 0,421 & 0,004 \\
\hline & & Ciudad_País + Jardín Botánico Las Orquídeas & 153,438 & 27 & 0,000 & 0,534 & 0,000 \\
\hline & & Género + Dique del Río Pambay & 1,903 & 1 & 0,017 & 0,701 & 0,017 \\
\hline & & Edad + Dique del Río Pambay & 16,190 & 5 & 0,006 & 0,420 & 0,006 \\
\hline & & Ciudad_País + Dique del Río Pambay & 96,042 & 27 & 0,000 & 0,447 & 0,000 \\
\hline & & Género + Dique Las Palmas & 17,132 & 1 & 0,000 & 0,421 & 0,000 \\
\hline & & Edad + Dique Las Palmas & 6,203 & 5 & 0,029 & 0,426 & 0,029 \\
\hline & & Ciudad_País + Dique Las Palmas & 69,950 & 27 & 0,000 & 0,420 & 0,000 \\
\hline & & Género + Parque Real de Aves Exóticas & 8,442 & 1 & 0,004 & 0,465 & 0,004 \\
\hline & & Edad + Parque Real de Aves Exóticas & 6,932 & 5 & 0,023 & 0,433 & 0,023 \\
\hline \multirow[b]{4}{*}{1} & & Ciudad_País + Parque Real de Aves Exóticas & 45,620 & 27 & 0,014 & 0,433 & 0,014 \\
\hline & & Género + Otro & 0,220 & 1 & 0,006 & 0,401 & 0,006 \\
\hline & & Edad + Otro & 20,946 & 5 & 0,001 & 0,427 & 0,001 \\
\hline & & Ciudad_País + Otro & 122,928 & 27 & 0,000 & 0,492 & 0,000 \\
\hline
\end{tabular}


Tabla 2: Resultados prueba Chi-cuadrado y Coeficiente de contingencia, datos de Julio 2018

\begin{tabular}{|c|c|c|c|c|c|c|c|}
\hline \multicolumn{8}{|c|}{ JULIO 2018} \\
\hline \multirow[b]{2}{*}{$\mathbf{N}^{\circ}$} & \multirow{2}{*}{$\begin{array}{l}\text { Combinación } \\
\text { de variables }\end{array}$} & \multirow[b]{2}{*}{ Variables comparadas } & \multicolumn{3}{|c|}{ Chi-cuadrado de Pearson } & \multicolumn{2}{|c|}{$\begin{array}{l}\text { Coeficiente de } \\
\text { contingencia }\end{array}$} \\
\hline & & & Valor & Gl & $\begin{array}{c}\text { Sig. } \\
\text { asintótica } \\
(2 \text { caras })\end{array}$ & Valor & $\begin{array}{l}\text { Aprox. } \\
\text { Sig. }\end{array}$ \\
\hline & \multirow{36}{*}{$\begin{array}{l}\text { Género + Edad } \\
+\quad \text { Ciudad_País } \\
+\quad \text { Atractivo } \\
\text { turístico }\end{array}$} & Género + Parque Acuático Morete Puyu & 0,729 & 1 & 0,039 & 0,435 & 0,039 \\
\hline & & Edad + Parque Acuático Morete Puyu & 7,411 & 5 & 0,019 & 0,437 & 0,019 \\
\hline & & Ciudad_País + Parque Acuático Morete Puyu & 60,588 & 37 & 0,009 & 0,409 & 0,009 \\
\hline & & Género + Parque Central Doce de Mayo & 0,456 & 1 & 0,045 & 0,440 & 0,045 \\
\hline & & Edad + Parque Central Doce de Mayo & 8,842 & 5 & 0,012 & 0,498 & 0,012 \\
\hline & & Ciudad_País + Parque Central Doce de Mayo & 63,130 & 37 & 0,005 & 0,438 & 0,005 \\
\hline & & Género + Museo Etnoarqueológico de Pastaza & 0,104 & 1 & 0,047 & 0,416 & 0,047 \\
\hline & & Edad + Museo Etnoarqueológico de Pastaza & 14,505 & 5 & 0,013 & 0,450 & 0,013 \\
\hline & & Ciudad_País + Museo Etnoarqueológico de Pastaza & 73,512 & 37 & 0,000 & 0,401 & 0,000 \\
\hline & & Género + Refugio de Vida Silvestre Yana Cocha & 0,001 & 1 & 0,048 & 0,466 & 0,048 \\
\hline & & Edad + Refugio de Vida Silvestre Yana Cocha & 20,103 & 5 & 0,001 & 0,422 & 0,001 \\
\hline & & Ciudad_País + Refugio de Vida Silvestre Yana Cocha & 76,828 & 37 & 0,000 & 0,408 & 0,000 \\
\hline & & Género + Paseo turístico del Río Puyo & 0,025 & 1 & 0,049 & 0,480 & 0,049 \\
\hline & & Edad + Paseo turístico del Río Puyo & 12,973 & 5 & 0,024 & 0,418 & 0,024 \\
\hline & & Ciudad_País + Paseo turístico del Río Puyo & 80,034 & 37 & 0,000 & 0,415 & 0,000 \\
\hline & & Género + Malecón Boayaku Puyu & 2,181 & 1 & 0,014 & 0,470 & 0,014 \\
\hline & & Edad + Malecón Boayaku Puyu & 2,802 & 5 & 0,030 & 0,450 & 0,030 \\
\hline & & Ciudad_País + Malecón Boayaku Puyu & 70,005 & 37 & 0,001 & 0,402 & 0,001 \\
\hline & & Género + Parque Etnobotánico Omaere & 3,604 & 1 & 0,048 & 0,463 & 0,048 \\
\hline & & Edad + Parque Etnobotánico Omaere & 10,302 & 5 & 0,047 & 0,432 & 0,047 \\
\hline & & Ciudad_País + Parque Etnobotánico Omaere & 79,354 & 37 & 0,000 & 0,413 & 0,000 \\
\hline & & Género + Jardín Botánico Las Orquídeas & 11,383 & 1 & 0,001 & 0,469 & 0,001 \\
\hline & & Edad + Jardín Botánico Las Orquídeas & 4,337 & 5 & 0,020 & 0,406 & 0,020 \\
\hline & & Ciudad_País + Jardín Botánico Las Orquídeas & 53,484 & 37 & 0,039 & 0,419 & 0,039 \\
\hline & & Género + Dique del Río Pambay & 0,089 & 1 & 0,026 & 0,452 & 0,026 \\
\hline & & Edad + Dique del Río Pambay & 12,382 & 5 & 0,030 & 0,418 & 0,030 \\
\hline & & Ciudad_País + Dique del Río Pambay & 56,304 & 37 & 0,022 & 0,407 & 0,022 \\
\hline & & Género + Dique Las Palmas & 0,447 & 1 & 0,038 & 0,405 & 0,038 \\
\hline & & Edad + Dique Las Palmas & 14,432 & 5 & 0,013 & 0,402 & 0,013 \\
\hline & & Ciudad_País + Dique Las Palmas & 52,538 & 37 & 0,047 & 0,407 & 0,047 \\
\hline & & Género + Parque Real de Aves Exóticas & 0,420 & 1 & 0,017 & 0,433 & 0,017 \\
\hline & & Edad + Parque Real de Aves Exóticas & 5,467 & 5 & 0,036 & 0,412 & 0,036 \\
\hline & & Ciudad_País + Parque Real de Aves Exóticas & 35,236 & 37 & 0,045 & 0,403 & 0,045 \\
\hline & & Género + Otro & 0,070 & 1 & 0,017 & 0,413 & 0,017 \\
\hline & & Edad + Otro & 2,374 & 5 & 0,038 & 0,428 & 0,038 \\
\hline 1 & & Ciudad_País + Otro & 74,627 & 37 & 0,000 & 0,403 & 0,000 \\
\hline
\end{tabular}


Tabla 3: Resultados prueba Chi-cuadrado y Coeficiente de contingencia, datos de agosto 2018

\begin{tabular}{|c|c|c|c|c|c|c|c|}
\hline \multicolumn{8}{|c|}{ AGOSTO 2018} \\
\hline \multirow[b]{2}{*}{$\mathbf{N}^{\circ}$} & \multirow[b]{2}{*}{$\begin{array}{c}\text { Combinación } \\
\text { de variables }\end{array}$} & \multirow[b]{2}{*}{ Variables comparadas } & \multicolumn{3}{|c|}{$\begin{array}{c}\text { Chi-cuadrado de } \\
\text { Pearson }\end{array}$} & \multicolumn{2}{|c|}{$\begin{array}{c}\text { Coeficiente de } \\
\text { contingencia }\end{array}$} \\
\hline & & & Valor & Gl & \begin{tabular}{|c|} 
Sig. \\
asintótica \\
$(2$ caras $)$
\end{tabular} & Valor & $\begin{array}{l}\text { Aprox. } \\
\text { Sig. }\end{array}$ \\
\hline & \multirow{36}{*}{$\begin{array}{l}\text { Género + } \\
\text { Edad } \\
\text { Ciudad_País } \\
+\quad \text { Atractivo } \\
\text { turístico }\end{array}$} & Género + Parque Acuático Morete Puyu & 1,328 & 1 & 0,025 & 0,586 & 0,025 \\
\hline & & Edad + Parque Acuático Morete Puyu & 10,046 & 5 & 0,039 & 0,416 & 0,039 \\
\hline & & Ciudad_País + Parque Acuático Morete Puyu & 70,617 & 49 & 0,023 & 0,404 & 0,023 \\
\hline & & Género + Parque Central Doce de Mayo & 0,161 & 1 & 0,047 & 0,420 & 0,047 \\
\hline & & Edad + Parque Central Doce de Mayo & 1,528 & 5 & 0,010 & 0,429 & 0,010 \\
\hline & & Ciudad_País + Parque Central Doce de Mayo & 51,065 & 49 & 0,039 & 0,422 & 0,039 \\
\hline & & Género + Museo Etnoarqueológico de Pastaza & 0,141 & 1 & 0,043 & 0,412 & 0,043 \\
\hline & & Edad + Museo Etnoarqueológico de Pastaza & 4,199 & 5 & 0,021 & 0,410 & 0,021 \\
\hline & & Ciudad_País + Museo Etnoarqueológico de Pastaza & 85,272 & 49 & 0,001 & 0,426 & 0,001 \\
\hline & & Género + Refugio de Vida Silvestre Yana Cocha & 0,030 & 1 &, 029 & 0,409 &, 029 \\
\hline & & Edad + Refugio de Vida Silvestre Yana Cocha & 1,758 & 5 & 0,015 & 0,429 & 0,015 \\
\hline & & Ciudad_País + Refugio de Vida Silvestre Yana Cocha & 79,387 & 49 & 0,004 & 0,413 & 0,004 \\
\hline & & Género + Paseo turístico del Río Puyo & 1,798 & 1 & 0,018 & 0,417 & 0,018 \\
\hline & & Edad + Paseo turístico del Río Puyo & 9,634 & 5 & 0,029 & 0,416 & 0,029 \\
\hline & & Ciudad_País + Paseo turístico del Río Puyo & 54,194 & 49 & 0,028 & 0,413 & 0,028 \\
\hline & & Género + Malecón Boayaku Puyu & 0,936 & 1 & 0,033 & 0,424 & 0,033 \\
\hline & & Edad + Malecón Boayaku Puyu & 2,977 & 5 & 0,036 & 0,409 & 0,036 \\
\hline & & Ciudad_País + Malecón Boayaku Puyu & 64,365 & 49 & 0,042 & 0,438 & 0,042 \\
\hline & & Género + Parque Etnobotánico Omaere & 1,342 & 1 & 0,025 & 0,406 & 0,025 \\
\hline & & Edad + Parque Etnobotánico Omaere & 7,917 & 5 & 0,016 & 0,419 & 0,016 \\
\hline & & Ciudad_País + Parque Etnobotánico Omaere & 90,256 & 49 & 0,000 & 0,436 & 0,000 \\
\hline & & Género + Jardín Botánico Las Orquídeas & 6,557 & 1 & 0,010 & 0,413 & 0,010 \\
\hline & & Edad + Jardín Botánico Las Orquídeas & 6,178 & 5 & 0,029 & 0,426 & 0,029 \\
\hline & & Ciudad_País + Jardín Botánico Las Orquídeas & 92,588 & 49 & 0,000 & 0,440 & 0,000 \\
\hline & & Género + Dique del Río Pambay & 0,296 & 1 & 0,012 & 0,428 & 0,012 \\
\hline & & Edad + Dique del Río Pambay & 10,152 & 5 & 0,010 & 0,416 & 0,010 \\
\hline & & Ciudad_País + Dique del Río Pambay & 60,571 & 49 & 0,012 & 0,404 & 0,012 \\
\hline & & Género + Dique Las Palmas & 0,043 & 1 & 0,035 & 0,411 & 0,035 \\
\hline & & Edad + Dique Las Palmas & 4,959 & 5 & 0,042 & 0,411 & 0,042 \\
\hline & & Ciudad_País + Dique Las Palmas & 36,971 & 49 & 0,048 & 0,403 & 0,048 \\
\hline & & Género + Parque Real de Aves Exóticas & 0,563 & 1 & 0,045 & 0,438 & 0,045 \\
\hline & & Edad + Parque Real de Aves Exóticas & 8,158 & 5 & 0,015 & 0,440 & 0,015 \\
\hline & & Ciudad_País + Parque Real de Aves Exóticas & 40,758 & 49 & 0,028 & 0,409 & 0,028 \\
\hline & & Género + Otro & 0,605 & 1 & 0,044 & 0,420 & 0,044 \\
\hline & & Edad + Otro & 15,087 & 5 & 0,010 & 0,419 & 0,010 \\
\hline 1 & & Ciudad_País + Otro & 76,919 & 49 & 0,007 & 0,408 & 0,007 \\
\hline
\end{tabular}


Las tablas anteriores muestran que todas las variables resultaron altamente relacionadas entre sí para los tres meses considerados (junio, julio y agosto 2018) debido a que reflejaron un valor de significación inferior a 0.05 , por lo cual se rechaza la hipótesis nula Ho, considerando las siguientes hipótesis:

H0: las variables son independientes (no están relacionadas entre sí).

H1: las variables son dependientes (si están relacionadas entre sí).

Por otro lado, los resultados del coeficiente de contingencia confirman la existencia de relación estadísticamente significativa entre todas las variables analizadas ya que sus valores están por encima de 0.40, según los criterios de Florián (2014) quien plantea que un valor de este coeficiente superior a 0.30 se considera que los indicadores o variables están fuertemente relacionados entre sí. El coeficiente de contingencia es una medida de relación estadística y expresa la intensidad de dicha relación entre dos o más variables nominales u ordinales.

Los resultados obtenidos sobre los indicadores analizados en base a la demanda turística, permiten conocer el Perfil del Visitante a la ciudad de Puyo, que se resume de la siguiente manera: en los tres meses se nota una mayor visita por parte del género masculino. En cuanto a la edad de los visitantes se observa que en el mes de junio en su mayoría están entre las edades de 35 a 44, en julio de 25 a 34 y en agosto entre 18 y 24. Se identifica como el principal sitio de procedencia para el mes de junio a Ambato, en el mes de julio se destacan Quito y Ambato y en agosto se mantiene Ambato.

En los tres meses se evidencia un gasto promedio de hasta 200 USD por la visita a Puyo. Se muestra que los viajeros en el mes de junio decidieron su visita luego de referencias de internet mientras que en los meses de julio y agosto lo hicieron por consultas a familiares. En los tres meses se refleja que el motivo de visita es por disponer de tiempo por vacaciones o feriado, seguido por el motivo mismo de hacer turismo, posteriormente descanso que se repite en los 3 meses. En los tres meses de estudio las encuestas reflejan como el sitio más visitado al Parque Acuático Morete Puyu, seguido del Dique del Río Pambay. Se muestra en los resultados obtenidos que cuando el viajero hace referencia a la ciudad de Puyo, su imaginario hace relación a la naturaleza en los tres meses. Los resultados de la encuesta reflejan que los visitantes en su mayoría pernoctan donde familiares o amigos en los meses de junio y julio, mientras que en el mes de agosto hacen uso de hostales y familiares o amigos en igual proporción.

Se diseñó el primer boletín (Figura 2), a partir de la demanda turística de cada mes y se inició el proceso de publicación de manera impresa y digital. Con este boletín se logró el funcionamiento del programa de la demanda turística que tendrá un seguimiento trimestral, considerando las últimas actualizaciones de visitantes. 
Figura 2. Portada del primer Boletín Informativo Trimestral (BIT-OT-UEA)

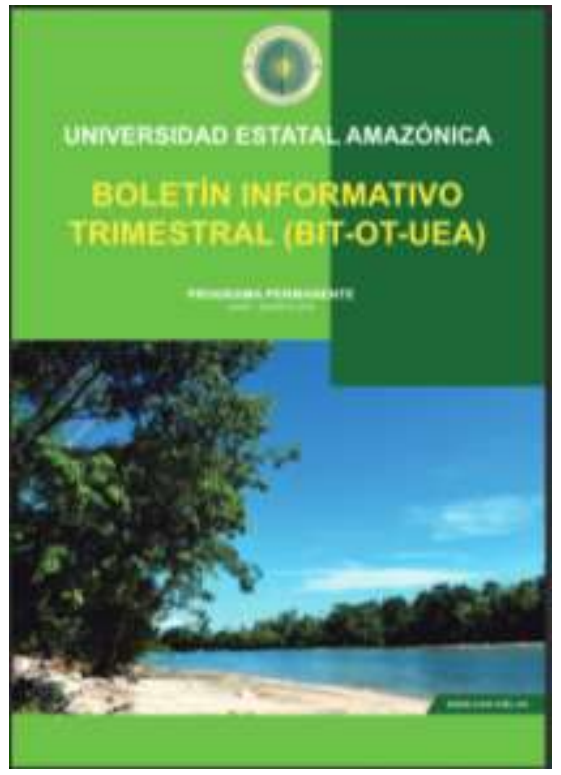

Finalmente, el OT-UEA se encargará de institucionalizar este programa para convertirlo en un referente de la información estadística y posteriormente posicionarse como un instrumento cuantitativo de medición, monitoreo y evaluación del comportamiento y tendencias de la demanda turística que estará disponible para toda la colectividad. Su funcionamiento se realiza en base a las variables que requieren los diferentes actores turísticos y considerando que el turismo es un sector de crecimiento acelerado es necesario disponer de información confiable que sea una verdadera herramienta de gestión. Actualmente, se han identificado un total de 98 observatorios turísticos, distribuidos en 27 países (sólo el $77 \%$ están en funcionamiento), mismos que son organismos importantes ya que sustentan la toma de decisiones tanto de la oferta como la demanda (Blasco \& Cuevas, 2013; Molina \& Báez, 2017).

\section{Conclusiones}

- Los resultados mostraron la demanda turística en Puyo (perfil del visitante) a partir de las variables más significativas: género, edad, ciudad_país, atractivo turístico, gasto turístico, ingresos, medios de información, motivo de visita, noches de estadía, idea_ciudad, tiempo de planificación, tipo de alojamiento, agencia de viaje, sitio después de la visita y sitio antes de la visita.

- Se estableció que las instituciones que tienen competencia dentro del turismo carecen de información de la demanda turística.

- Se diseñó e implementó el programa permanente el cual permite conocer la demanda turística para la toma de decisiones de los actores turísticos, tomando de referencia la estructura organizativa del modelo de gestión del OT-UEA. 
- Se elaboró el primer Boletín Informativo OT-UEA 2018 para su difusión, que servirá de instrumento para la toma de decisiones de los actores dentro del sistema turístico y el mismo se actualizará permanentemente en períodos trimestrales.

\section{Referencias Bibliográficas}

Ascanio, A. (2012). Teoría del turismo. México: Editorial Trillas.

Blasco, D., \& Cuevas, T. (2013). Observatorio en Turismo: Organismo inteligente para la toma de decisiones en el destino. Revista Iberoamericana de Turismo - RITUR, 2534.

Boullón, R. (2006). Planificación del espacio turístico. México: Editorial Trillas.

Centro de Estudios Superiores en Turismo. (2012). www.gob.mx/sectur. Obtenido de www.gob.mx/sectur:

file:///C:/Users/pc/Desktop/PCT-

UEA\%201.0/MARCO\%20TEORICO/sectur.pdf

Florián, C. (21/01/2014). www.prezi.com. Obtenido de www.prezi.com: https://prezi.com/3hf-etjmkdj/coeficiente-de-contingencia/

George, D., \& Mallery, P. (2003). SPSS for Windows step by step: A simple guide and reference. 11.0. Boston: Allyn \& Bacon.

Ministerio de Turismo. (19/11/2015). www.turismo.gob.ec. Obtenido de Www.turismo.gob.ec: file://C:/Users/pc/Desktop/PCTUEA\%201.0/MARCO\%20TEORICO/Mintur\%20implement\%C3\%B3\%20Cuenta $\% 20$ Sat $\%$ C3\%A9lite $\% 20 \mathrm{de} \% 20$ Turismo\%20\%E2\%80\%93\%20Ministerio\%20de\% 20Turismo.htm\#

Molina, E., \& Báez, S. (2017). Los Observatorios Turísticos a través de los tiempos . Revista Turydes: Turismo y Desarrollo, n. 22 (junio 2017).

Organización Mundial del Turismo. (2017). http://cf.cdn.unwto.org. Obtenido de http://cf.cdn.unwto.org: http://cf.cdn.unwto.org/sites/all/files/pdf/annual_report_2016_web_0.pdf

Rangel, E. (2013). Observatorio Turístico - Universidad Estatal Amazónica. Puyo.

Universidad Estatal Amazónica. (2018). uea.edu.ec. Obtenido de uea.edu.ec: https://www.uea.edu.ec/index.php/inicio/quienes-somos

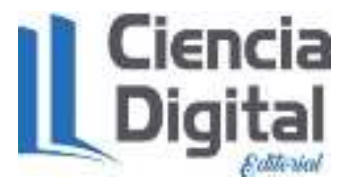




\section{PARA CITAR EL ARTÍCULO INDEXADO.}

Pinta Rodríguez, M., Chávez Esponda, D., \& Ruiz Mármol, E. (2019). Programa permanente de análisis de la demanda turística del Puyo, observatorio turístico de la Universidad Estatal Amazónica. Ciencia Digital, 3(3), 374-385. https://doi.org/10.33262/cienciadigital.v3i3.651

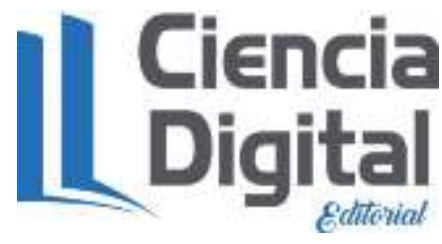

El artículo que se publica es de exclusiva responsabilidad de los autores y no necesariamente reflejan el pensamiento de la Revista Ciencia Digital.

El artículo queda en propiedad de la revista y, por tanto, su publicación parcial y/o total en otro medio tiene que ser autorizado por el director de la Revista Ciencia Digital.
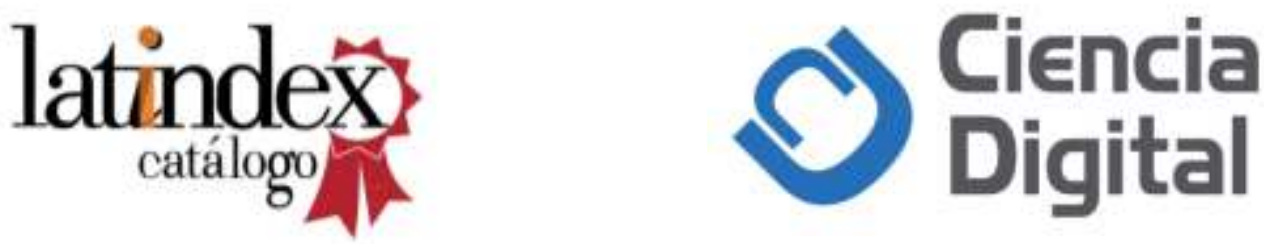